\title{
Inhibition of triggering receptor expressed on myeloid cells-1 ameliorates experimental autoimmune neuritis
}

\author{
XIAOLIANG ZHOU ${ }^{1,2^{*}}$, YANLI WANG $^{3 *}$, YUZHONG WANG $^{4}$, PETER CIPRIANO $^{2}$, BO XIAO $^{1}$ and WENBIN ZHOU $^{1}$ \\ ${ }^{1}$ Department of Neurology, Xiangya Hospital, Central-South University, Changsha, \\ Hunan 410008, P.R. China; ${ }^{2}$ Department of Radiology, Stanford Medical Center, Stanford, CA 94305, USA; \\ ${ }^{3}$ Department of Neurology, Jingzhou Central Hospital, Jingzhou, Hubei 434020; ${ }^{4}$ Department of \\ Neurology, Affiliated Hospital of Jining Medical University, Jining, Shandong 272000, P.R. China
}

Received November 29, 2015; Accepted December 15, 2016

DOI: $10.3892 / \mathrm{mmr} .2017 .6167$

\begin{abstract}
Experimental autoimmune neuritis (EAN) is a cluster of differentiation $4^{+} \mathrm{T}$ helper 1 cell-mediated inflammatory demyelinating disease of the peripheral nervous system and serves as a useful animal model for Guillain-Barré syndrome. Triggering receptor expressed on myeloid cells-1 (TREM-1) is an important receptor involved in sepsis and the innate inflammatory response. Linear plasmid 17 (LP 17) peptide is a competitive antagonist of TREM-1. To investigate the role of TREM-1 in EAN, 64 male Lewis rats were randomly divided into four groups: Normal saline, complete Freund's adjuvant, EAN and LP 17. The present study assessed the mRNA expression levels of TREM-1, tumor necrosis factor- $\alpha$ and interleukin-1 $\beta$ in sciatic nerves and peripheral blood mononuclear cells. The results demonstrated that inhibiting TREM-1 by administering LP 17 ameliorated symptoms and reduced inflammation in EAN rats. The present study concluded that TREM-1 may be involved in the pathogenesis of EAN, and that inhibition of TREM-1 may ameliorate EAN.
\end{abstract}

Correspondence to: Professor Wenbin Zhou, Department of Neurology, Xiangya Hospital, Central-South University, 87 Xiangya Road, Changsha, Hunan 410008, P.R. China

E-mail: zhouwenbin64@sina.cn

*Contributed equally

Abbreviations: EAN, experimental autoimmune neuritis; TREM-1, triggering receptor expressed on myeloid cells-1; PNS, peripheral nervous system; GBS, Guillain-Barré syndrome; DAP12, DNAX-activation protein of $12 \mathrm{kDa}$; PBMCs, peripheral blood mononuclear cells; NS, normal saline; CFA, complete Freund's adjuvant; FIA, Freund's incomplete adjuvant; PI, post-immunization; H\&E, hematoxylin and eosin; RT-qPCR, reverse transcription-quantitative polymerase chain reaction

Key words: experimental autoimmune neuritis, triggering receptor expressed on myeloid cells-1, linear plasmid 17 peptide, interleukin- $1 \beta$, tumor necrosis factor- $\alpha$

\section{Introduction}

Experimental autoimmune neuritis (EAN) is a cluster of differentiation (CD) $4^{+} \mathrm{T}$ cell-mediated inflammatory demyelinating disease of the peripheral nervous system (PNS) (1). EAN serves as a useful animal model for Guillain-Barré syndrome (GBS), which is a $\mathrm{CD}^{+} \mathrm{T}$ cell-mediated disease of the PNS in humans (2).

Triggering receptor expressed on myeloid cells-1 (TREM-1) is a $30-\mathrm{kDa}$ glycoprotein associated with DNAX-activation protein of $12 \mathrm{kDa}$ (DAP12), which is an immunoreceptor tyrosine-based activation motif-containing adaptor protein (3). TREM-1 is selectively expressed on blood neutrophils and on a subset of monocytes $(4,5)$ and may amplify the inflammatory response (5-7). TREM-1 induces the production of chemokines and cytokines via DAP12 (3). TREM-1 additionally regulates signaling pathways induced by known classes of pattern-recognition receptors, including Toll-like receptors and neuronal apoptosis inhibitory proteins (4). Previous studies have demonstrated that TREM-1 is associated with numerous types of autoimmune diseases, including inflammatory bowel diseases (8), acute pancreatitis (9), neoplastic pleural effusions (10) and rheumatoid arthritis (11). TREM-1 influences chronic heart rejection by regulating the infiltration and differentiation of CD4 ${ }^{+}$lymphocytes (12). In addition, TREM-1 has a tumor promoting effect (13).

Although TREM-1 has been widely studied, its role in EAN/GBS remains to be investigated. To determine whether TREM-1 is involved in the pathogenesis of EAN, the present study treated the animal model of EAN with linear plasmid 17 (LP 17), an analogue synthetic peptide derived from the extracellular moiety of TREM-1 (14). The symptoms of rats and the mRNA expression levels of tumor necrosis factor (TNF)- $\alpha$, interleukin (IL)- $1 \beta$ and TREM-1 in sciatic nerves and peripheral blood mononuclear cells (PBMCs) were subsequently assessed. The results of the present study suggested that TREM-1 may serve a role in the pathogenesis of EAN.

\section{Materials and methods}

Animal models. Animal procedures were approved by Xiangya Hospital Ethics Committee, Xiangya Hospital (Changsha, 
China). The Guidelines for Ethical Conduct in the Care and Use of Vertebrate Animals in Research and Training by the American Physiological Society Council were followed (15).

Specific pathogen free male Lewis rats (age, 6-8 weeks; weight, 160-180 g; n=64) were purchased from Vital River Laboratories Co., Ltd. (Beijing, China) and were housed in groups of two or three per cage under controlled conditions: Light-dark cycle, 12-h; background noise, $40 \pm 10 \mathrm{~dB}$; relative humidity, 50-60\%; temperature, $20 \pm 3^{\circ} \mathrm{C}$; and food and water ad libitum. Rats were acclimatized for one week, following which they were randomly assigned to four groups: Normal saline (NS), complete Freund's adjuvant (CFA), EAN and LP 17.

Myelin P2 peptides 53-78 (THR-GLU-SER-PRO-PHE-L YS-ASN-THR-GLU-ILE-SER-PHE-LYS-LEU-GLY-GLN-G LU-PHE-GLU-GLU-THR-THR-ALA-ASP-ASN-ARG) were synthesized by solid-phase stepwise elongation using a Tecan peptide synthesizer (GL Biochem Ltd., Shanghai, China).

The EAN animal model was created by injecting the two hind-foot pads with $200 \mu \mathrm{l}$ antigen emulsion containing $100 \mu \mathrm{g}$ P2 53-78 emulsified in $100 \mu \mathrm{l}$ saline and $100 \mu \mathrm{l}$ Freund's incomplete adjuvant (FIA; Sigma-Aldrich; Merck Millipore, Darmstadt, Germany) containing $5 \mathrm{mg} / \mathrm{ml}$ heat-inactivated Mycobacterium tuberculosis H37RA (Beijing Institute of Biological Products, Co., Ltd., Beijing, China) (16). The FIA + Mycobacterium tuberculosis H37RA mixture was referred to as CFA.

Rats in the NS group were injected with $200 \mu 1$ sterile saline, and rats in the CFA group with $200 \mu \mathrm{l} \mathrm{CFA.} \mathrm{The} \mathrm{NS}$ and CFA groups served as controls. Rats in the LP 17 group were injected with $200 \mu \mathrm{l}$ antigen emulsion and a single dose (4 mg/kg, dissolved in $500 \mu \mathrm{l} \mathrm{NS}$ and injected intraperitoneally) of LP 17 (GL Biochem Ltd., Shanghai, China) administered immediately following the footpad injection.

Body weight and clinical scores were assessed immediately prior to immunization and every day following immunization until 33 days post-immunization (PI). The timeline of the experiment was numbered as days PI. Severity of paresis was graded as follows: $0=$ normal; $1=$ flaccid tail; $2=$ moderate paraparesis; $3=$ severe paraparesis; $4=$ tetraparesis (16).

Sample collection. Each group contained four subgroups ( $n=4 /$ subgroup) that were sacrificed at different days PI: 7 (phase 1: Onset of disease), 16 (phase 2: Disease peak), 24 (phase 3: Early recovery phase) and 33 (phase 4: Late recovery phase). Prior to sacrifice, rats anesthetized with an intraperitoneal injection of $36 \mathrm{mg} / \mathrm{kg} 3 \%$ chloral hydrate (Xiangya Hospital, Changsha, China) to draw blood and to carefully separate the bilateral sciatic nerves (stored at $-80^{\circ} \mathrm{C}$ for mRNA analysis and collected for histopathological assessment, respectively), and then the anesthetized rats were sacrificed in a $0.0151 \mathrm{CO}_{2}$ gas chamber, where the rats received a $100 \% \mathrm{CO}_{2}$ air flow for $10 \mathrm{~min}$. Mortality was confirmed with no heartbeat/breath by at least two technicians (Xiangya Hospital, Changsha, China).

Blood samples $(\sim 5 \mathrm{ml})$ were obtained from the jugular vein and collected in EDTA-coated tubes. PBMCs were isolated by gradient centrifugation using LymphoPrep ${ }^{\mathrm{TM}}$ solution (Beijing Dingguo Changsheng Biotechnology Co., Ltd., Beijing, China), and the whole blood was centrifuged at $1,200 \times \mathrm{g}$ for $15 \mathrm{~min}$ at $4^{\circ} \mathrm{C}$. The buffy coat was mixed with RPMI-1640 (Sigma-Aldrich; Merck Millipore). The buffy coat/RPMI mix was layered on top of endotoxin-free LymphoPrep and centrifuged at $1,200 \mathrm{x} g$ for $25 \mathrm{~min}$ at $4^{\circ} \mathrm{C}$. PBMCs separated out into a distinct layer that was removed, washed twice with RPMI-1640 and centrifuged at $600 \mathrm{x} g$ for $10 \mathrm{~min}$ at $4^{\circ} \mathrm{C}$, then $400 \mathrm{xg}$ for $10 \mathrm{~min}$ at $4^{\circ} \mathrm{C}$. Following three washes with PBS, PBMCs were collected for RNA extraction.

Histopathological assessment of EAN. Sciatic nerves were isolated and fixed overnight in $4 \%$ paraformaldehyde at $4^{\circ} \mathrm{C}$. Each sciatic nerve was cut into segments $\sim 5-\mathrm{mm}$ long, which were embedded in paraffin blocks, sectioned serially $(4 \mu \mathrm{m})$, and mounted on polylysine-treated slides (Beijing Dingguo Changsheng Biotechnology Co., Ltd.). Hematoxylin and eosin (H\&E) staining was performed to observe inflammatory cell infiltration in the nerves $(17,18)$. This served to validate the EAN animal model.

Reverse transcription-quantitative polymerase chain reaction $(R T-q P C R)$. Total RNA was isolated from PBMCs and sciatic nerves using TRIzol ${ }^{\circledR}$ reagent (Invitrogen; Thermo Fisher Scientific, Inc., Waltham, MA, USA). RNA purity and concentration were confirmed by spectrophotometry with a wavelength of $450 \mathrm{~nm}$ using the NanoDrop ND-1000 Spectrophotometer (NanoDrop Technologies; Thermo Fisher Scientific, Inc.) cDNA was synthesized using a Reverse-Transcription kit (Toyobo Co., Ltd., Osaka, Japan) and an ABI StepOnePlus ${ }^{\mathrm{TM}}$ PCR system (Applied Biosystems; Thermo Fisher Scientific, Inc.).

Primer sequences were as follows: Forward, 5'-TTCAAC GGCACAGTCAAG-3' and reverse, 5'-CCAGCATCACCC CATTT-3' for TREM-1; forward, 5'-CCTGGTCACCAAATC AGCATTA-3' and reverse, 5'-GAAGCTGTCTTCAGGCCA ACAT-3' for TNF- $\alpha$; forward, 5'-ATGAGAGCATCCAGC TTCAAATC-3' and reverse, 5'-CACACTAGCAGGTCGTCA TCATC-3' for IL-1 $\beta$; and forward, 5'-TTCAACGGCACA GTCAAG-3' and reverse, 5'-CCAGCATCACCCCATTT-3' for GAPDH. The primers were synthesized by Sangon Biotech Co., Ltd. (Shanghai, China). cDNAs were amplified with SYBR Green using the Platinum SYBR-Green qPCR SuperMix UDG (Invitrogen; Thermo Fisher Scientific, Inc.). A RevertAid First Strand cDNA synthesis kit (Thermo Fisher Scientific, Inc.) and nuclease-free water (Thermo Fisher Scientific, Inc.) were also used during the process of qPCR.

Cycling conditions were identical for all primer pairs: An initial denaturation step at $95^{\circ} \mathrm{C}$ for $1 \mathrm{~min}$, followed by 40 cycles at $95^{\circ} \mathrm{C}$ for $15 \mathrm{sec}$ and $65^{\circ} \mathrm{C}$ for $1 \mathrm{~min}$. The results were automatically analyzed using the ABI StepOnePlus PCR system and the $2^{-\Delta \Delta \mathrm{Cq}}$ method was used to analyze mRNA expression $(\Delta \mathrm{Cq}$ represents the difference in quantification cycle value between the target gene and the internal control; $\Delta \Delta \mathrm{Cq}$ represents the difference in $\Delta \mathrm{Cq}$ between groups) (19). Three independent RT-qPCR reactions were performed.

Statistical analysis. Data are presented as the mean \pm standard deviation. Analyses were performed using SPSS software version 18.0 (SPSS, Inc., Chicago, IL, USA). Differences between groups were evaluated by one-way analysis of variance 
with Bonferroni post-test analyses. $\mathrm{P}<0.05$ was considered to indicate a statistically significant difference.

\section{Results}

Clinical and histopathological alterations. Rats in the EAN group $(n=16)$ appeared healthy prior to immunization and began to exhibit fatigue and tail weakness 9 to 11 days PI. They had the greatest clinical score $(2.44 \pm 0.21 ; n=12)$ on day 16 and then gradually recovered, with complete recovery by day $33(n=4)$. Severity of disease in the LP 17 group was reduced compared with the EAN group $(\mathrm{P}<0.05$; Fig. 1$)$; however, the clinical score of the LP 17 group also peaked on day 16 (1.57 $\pm 0.19 ; n=12$; Fig. 1$)$.

The infiltration of inflammatory cells in the LP 17 group (Fig. 2A) was markedly reduced compared with the EAN group (Fig. 2B), and fewer histopathological alterations were observed.

TREM-1 mRNA expression levels. In sciatic nerves (Fig. 3A) and PBMCs (Fig. 3B), mRNA expression levels of TREM-1 were increased across all phases in the EAN group compared with the CFA and NS groups $(\mathrm{P}<0.05)$. The expression levels of TREM-1 mRNA were markedly increased in sciatic nerves compared with PBMCs. In the PBMCs, mRNA expression levels of TREM-1 in the EAN and LP 17 groups differed significantly between the disease peak phase and early recovery phase $(\mathrm{P}<0.05)$. Compared with the control groups, mRNA expression levels of TREM-1 in PBMCs were increased in the LP 17 group at the onset of disease (day 7) and disease peak (day 16) phases $(\mathrm{P}<0.05)$.

TNF- $\alpha$ mRNA expression levels. In sciatic nerves (Fig. 4A) and PBMCs (Fig. 4B), mRNA expression levels of TNF- $\alpha$ were increased in the EAN group compared with the control and LP 17 groups across all phases $(\mathrm{P}<0.05)$, and there was a significant difference between each phase within the EAN group $(\mathrm{P}<0.05)$.

In sciatic nerves, the mRNA expression levels of TNF- $\alpha$ were significantly different across the first three phases within the LP 17 group $(\mathrm{P}<0.01)$. mRNA expression levels of TNF- $\alpha$ in the sciatic nerves of the LP 17 group were increased compared with the NS and CFA groups at disease peak $(\mathrm{P}<0.05)$ and were increased compared with the NS group in the early recovery phase $(\mathrm{P}<0.05)$. TNF- $\alpha$ mRNA levels were markedly increased in the EAN group compared with the LP 17 group $(\mathrm{P}<0.01)$.

In PBMCs, TNF- $\alpha$ mRNA expression levels were increased in the LP 17 group compared with the control groups in the first three phases $(\mathrm{P}<0.05)$, and there were significant differences between the EAN and LP 17 groups across all phases $(\mathrm{P}<0.05)$.

$I L-1 \beta$ mRNA expression levels. In sciatic nerves (Fig. 5A), mRNA expression levels of IL-1 $\beta$ were increased in the EAN group compared with the control and LP 17 groups across all phases $(\mathrm{P}<0.05)$, and were significantly different between each phase within the EAN group $(\mathrm{P}<0.05)$. IL-1 $\beta$ mRNA expression levels in the LP 17 group were increased compared with the control groups; however, were reduced compared with the EAN group across all phases $(\mathrm{P}<0.05)$.

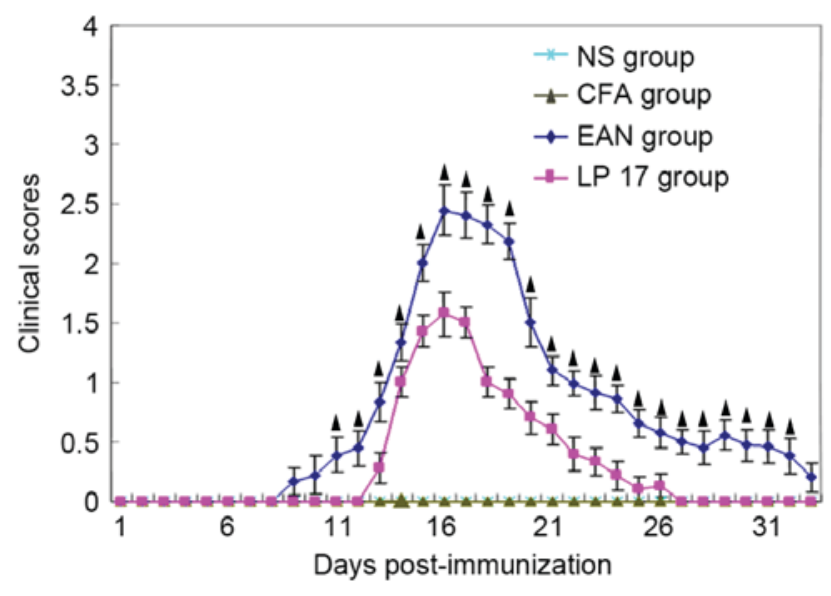

Figure 1. Clinical scores. EAN and LP 17 group rats demonstrated greater clinical scores compared with the control groups in the disease peak (day 16) and early recovery (day 24) phases. Rats in the EAN group had the greatest clinical score on day 16. Rats in the LP 17 group were less severely affected; however, similarly had a clinical score that peaked on day 16. Data are expressed as the mean \pm standard deviation. ${ }^{\boldsymbol{\Delta}} \mathrm{P}<0.05$ vs. all other groups. NS, normal saline; CFA, complete Freund's adjuvant; EAN, experimental autoimmune neuritis; LP 17, linear plasmid 17.

\section{A}

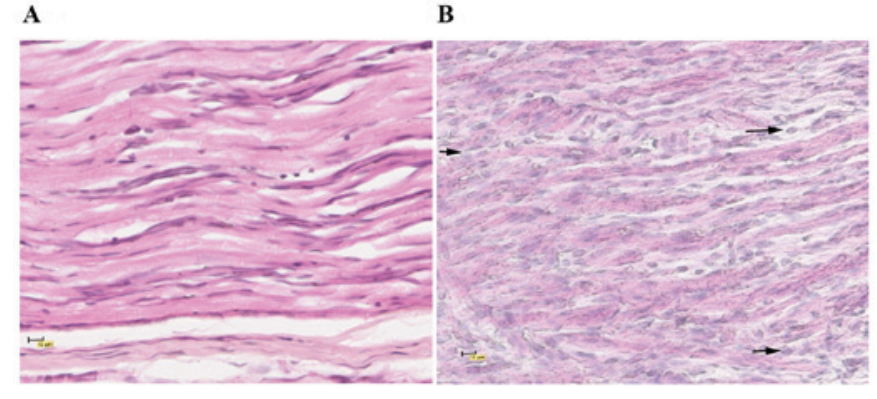

Figure 2. Hematoxylin and eosin staining of the sciatic nerve on day 16 Infiltration of inflammatory cells in (A) the LP 17 group was markedly reduced compared with (B) the EAN group, as indicated by the black arrows. Original magnification, $x 40$; scale bar $=10 \mu \mathrm{m}$. LP 17, linear plasmid 17; EAN, experimental autoimmune neuritis.

IL-1 $\beta$ mRNA expression levels in PBMCs (Fig. 5B) were increased in the EAN group compared with the LP 17 group at the disease peak and late recovery phases $(\mathrm{P}<0.05)$. Compared with the control groups, mRNA expression levels of IL-1 $\beta$ were increased in the EAN and LP 17 groups in the disease peak and early recovery phases $(\mathrm{P}<0.05)$.

In sciatic nerves and PBMCs, IL- $1 \beta$ mRNA expression levels were significantly different between the first three phases within the EAN and LP 17 groups $(\mathrm{P}<0.05)$.

\section{Discussion}

TREM-1 potently amplifies inflammatory responses by enhancing degranulation and secretion of proinflammatory mediators (8). Simultaneously, the activation of TREM-1 increases the expression levels of costimulatory molecules on the surface of mononuclear leucocytes (20).

Elevated TREM-1 expression levels may serve as an early indicator of poor prognosis. Previous studies have suggested that TREM-1 may represent a valuable marker for 

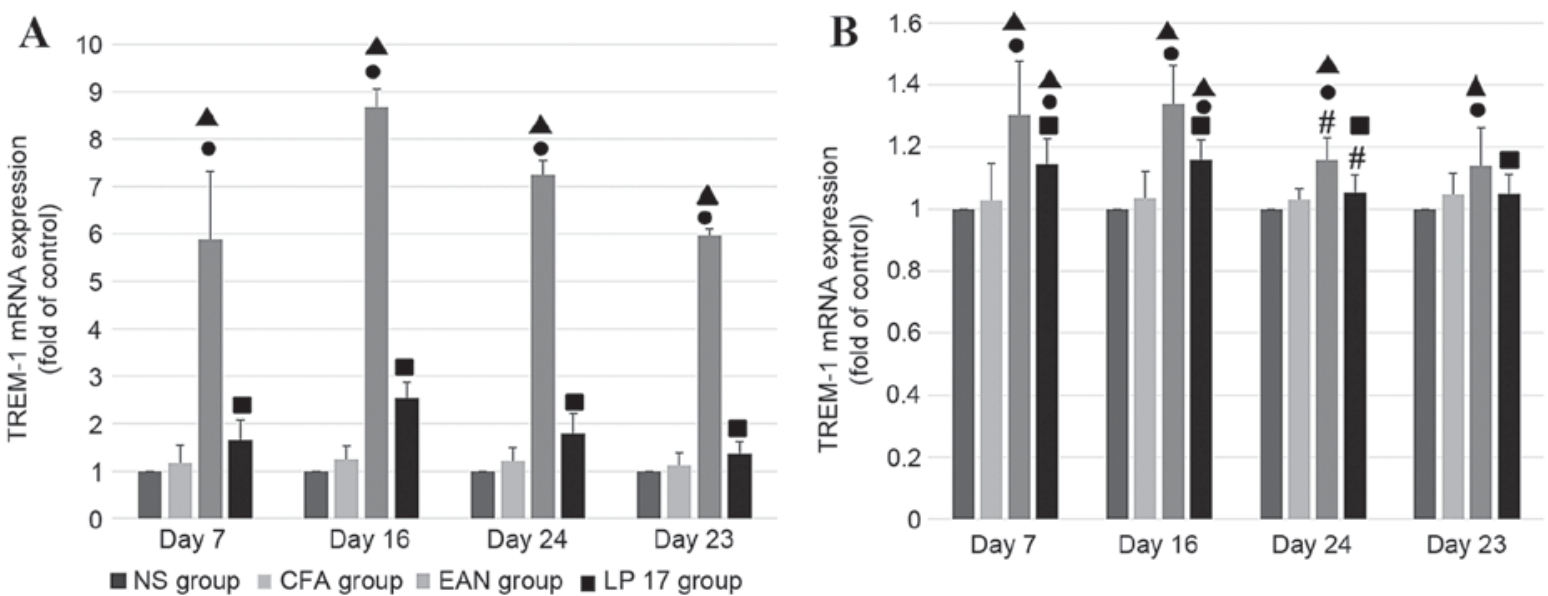

Figure 3. TREM-1 mRNA expression levels. TREM-1 mRNA expression levels in the EAN group were increased compared with all other groups in (A) sciatic nerves and (B) PBMCs, and were increased in sciatic nerves compared with PBMCs. Data are expressed as the mean \pm standard deviation. ${ }^{\boldsymbol{\Delta}} \mathrm{P}<0.05$ vs. CFA group; ${ }^{\circ} \mathrm{P}<0.05$ vs. NS group; ${ }^{\mathrm{P}}<0.05$ vs. EAN group; ${ }^{\#} \mathrm{P}<0.05$ vs. previous time point. NS, normal saline; CFA, complete Freund's adjuvant; EAN, experimental autoimmune neuritis; TREM-1, triggering receptor expressed on myeloid-cells 1; LP 17, linear plasmid 17.
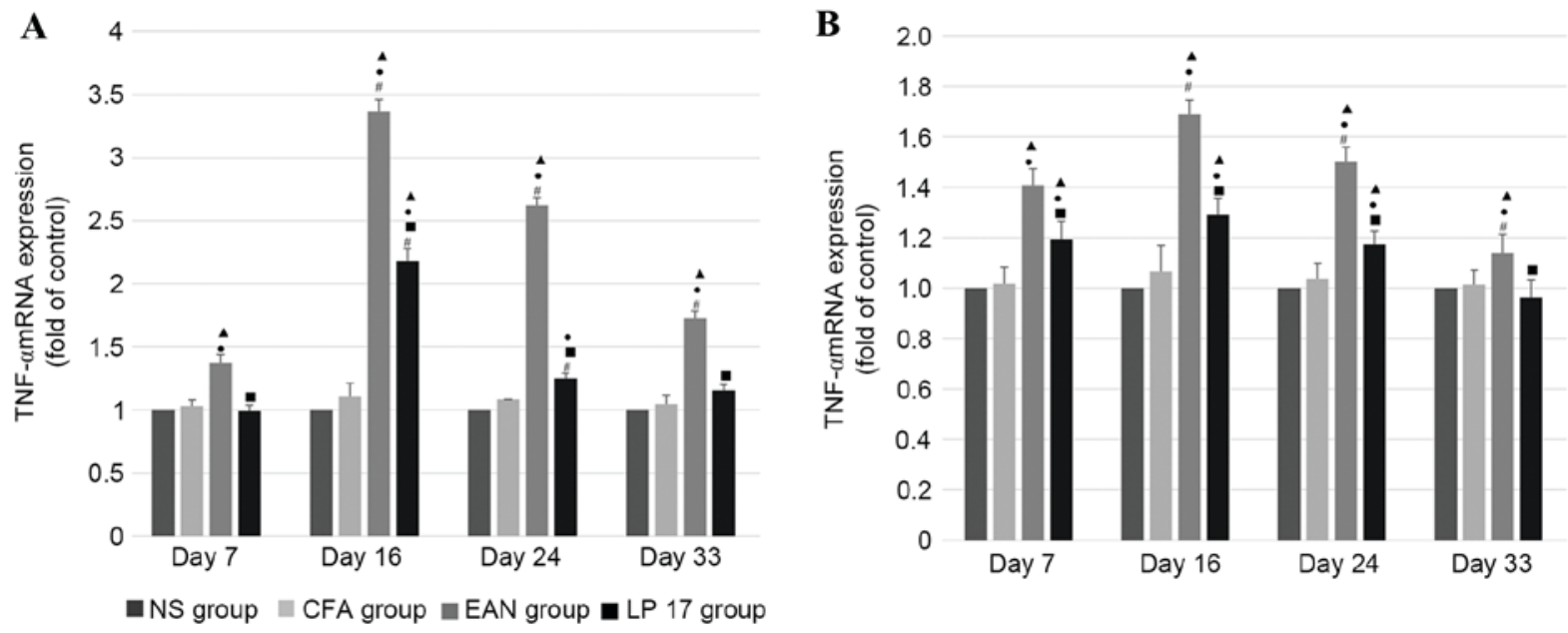

Figure 4. TNF- $\alpha$ mRNA expression levels. TNF- $\alpha$ mRNA expression levels in the EAN group were increased compared with the other groups in (A) sciatic nerves and (B) PBMCs, and were greatest on day 16 in the two groups. Data are expressed as the mean \pm standard deviation. ${ }^{\wedge} \mathrm{P}<0.05$ vs. CFA group; ${ }^{\bullet} \mathrm{P}<0.05$ vs. NS group; $" \mathrm{P}<0.05$ vs. EAN group; ${ }^{\#} \mathrm{P}<0.05$ vs. previous time point. NS, normal saline; $\mathrm{CFA}$, complete Freund's adjuvant; EAN, experimental autoimmune neuritis; LP 17, linear plasmid 17; TNF- $\alpha$; tumor necrosis factor- $\alpha$.

A

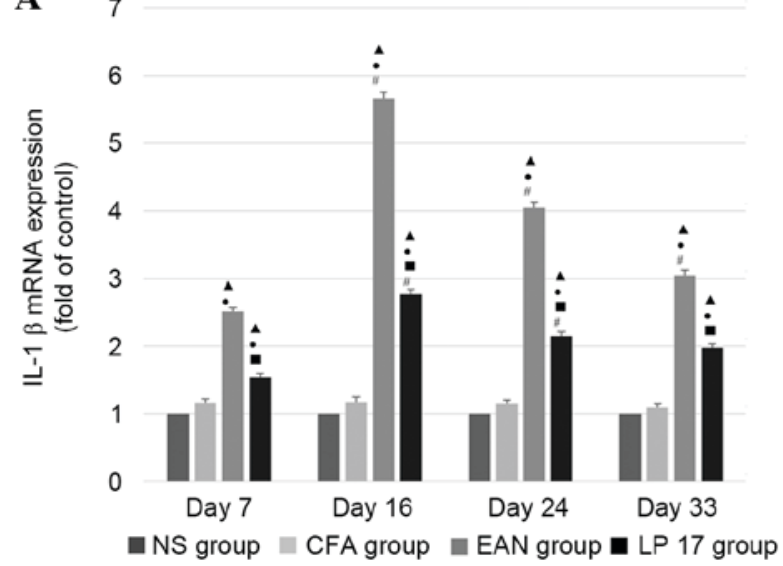

B

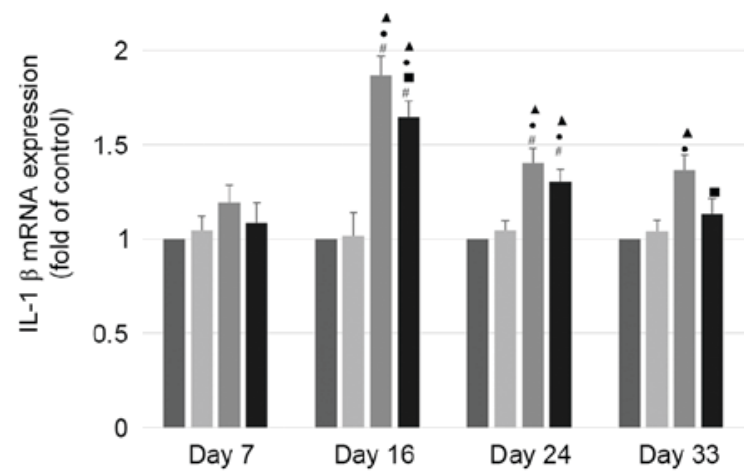

Figure 5. IL-1 $\beta$ mRNA expression levels. IL-1 $\beta$ mRNA expression levels in (A) sciatic nerves in the EAN group on day 16 were markedly increased compared with the other groups across all phases, and were greatest on day 16 in sciatic nerves and (B) PBMCs. Data are expressed as the mean \pm standard deviation. ${ }^{\wedge} \mathrm{P}<0.05$ vs. CFA group; ${ }^{\bullet} \mathrm{P}<0.05$ vs. NS group; ${ }^{-} \mathrm{P}<0.05$ vs. EAN group; ${ }^{*} \mathrm{P}<0.05$ vs. previous time point. NS, normal saline; CFA, complete Freund's adjuvant; EAN, experimental autoimmune neuritis; LP 17, linear plasmid 17; IL-1 $\beta$, interleukin-1 $\beta$. 
inflammatory severity and be a potential therapeutic target for the treatment of sepsis $(21,22)$.

In the present study, the mRNA expression levels of TREM-1 closely paralleled the clinical characteristics of the disease, and mRNA expression levels of TNF- $\alpha$, IL-1 $\beta$ and TREM-1 were greatest in the EAN group. Compared with the EAN group, mRNA expression levels of TREM-1 were markedly decreased in the LP 17 group, suggesting that LP 17 significantly inhibited the activation of the TREM-1 signaling pathway. Compared with PBMCs, TREM-1 mRNA expression levels were increased markedly in the sciatic nerve. These levels were elevated on day 7 in sciatic nerves and PBMCs, and peaked on day 16. This indicated that TREM-1 is involved in the early immune activation process of EAN.

As expected, rats in the LP 17 group experienced less weight loss and had reduced clinical scores compared with the EAN group. LP 17 did not completely inhibit EAN; however, the results of the present study demonstrated that LP 17 attenuates the symptoms of EAN in rats, indicating that TREM-1 is a potential target for the treatment of EAN.

Similarly, H\&E staining revealed that infiltration of inflammatory cells in sciatic nerves was less marked in the LP 17 group on day 16 compared with the EAN group. Therefore, TREM-1 may serve a role in a series of proinflammatory processes underlying EAN.

TNF- $\alpha$ may increase the permeability of the blood nerve barrier (20) and is involved in the pathogenesis of EAN in rats (21). Expression levels of TNF- $\alpha$ were greatest in the EAN group on day 7 (onset of disease); this is consistent with its role in the early pathological phase of EAN (22). In GBS, TNF- $\alpha$ produced by infiltrating $\mathrm{T}$ cells has a direct myelinotoxic effect (23), which may contribute to the increased expression levels of TNF- $\alpha$ in the sciatic nerves.

A previous study demonstrated that IL- $1 \beta$ mRNA is expressed in the lymph nodes and sciatic nerves of rats during the early onset phase of EAN, which may be associated with the infiltration of mononuclear macrophages (24). Similarly, the results of the present study revealed that the mRNA expression levels of IL-1 $\beta$ were increased in the sciatic nerves of the EAN group across all phases, and in the LP 17 group were greatest at day 16. A possible explanation is that IL-1 $\beta$, mediated by calpain, which is activated by calcium influx following nerve injury (25), was immediately and continuously released at the site of injury following injection of the antigen emulsion. In addition, peripheral inflammation may influence neurotransmitter metabolism, neuroendocrine function, as well as growth factor production, which modifies neural circuitry and may additionally account for the increased expression levels of IL-1 $\beta(25,26)$.

Although there are therapeutic benefits to the selective inhibition of TREM-1, a previous study has suggested that TREM-1 signaling is necessary for successful antimicrobial responses (24) and that inappropriate modulation of the TREM-1 signaling pathway may have profound and potentially detrimental effects on septic patients.

The present study demonstrates that TREM-1 is a potential therapeutic target for the treatment of GBS. Further studies involving expression and silencing of the TREM-1 gene are required to determine whether the protein itself is expressed, and which cells produce it.

\section{References}

1. Katzav A, Bina H, Aronovich R and Chapman J: Treatment for experimental autoimmune neuritis with clodronate (Bonefos). Immunol Res 56: 334-340, 2013.

2. Xu H, Li XL, Yue LT, Li H, Zhang M, Wang S, Wang CC and Duan RS: Therapeutic potential of atorvastatin-modified dendritic cells in experimental autoimmune neuritis by decreased Th1/Th17 cytokines and up-regulated T regulatory cells and NKR-P1(+) cells. J Neuroimmunol 269: 28-37, 2014.

3. Montalvo V, Quigley L, Vistica BP, Boelte KC, Nugent LF, Takai T, McVicar DW and Gery I: Environmental factors determine DAP12 deficiency to either enhance or suppress immunopathogenic processes. Immunology 140: 475-482, 2013.

4. Arts RJ, Joosten LA, van der Meer JW and Netea MG: TREM-1: Intracellular signaling pathways and interaction with pattern recognition receptors. J Leukoc Biol 93: 209-215, 2013.

5. Palazzo SJ, Simpson T and Schnapp LM: Triggering receptor expressed on myeloid cells type 1 as a potential therapeutic target in sepsis. Dimens Crit Care Nurs 31: 1-6, 2012.

6. Bouchon A, Facchetti F, Weigand MA and Colonna M: TREM-1 amplifies inflammation and is a crucial mediator of septic shock. Nature 410: 1103-1107, 2001.

7. Zangi L, Klionsky YZ, Yarimi L, Bachar-Lustig E, Eidelstein Y, Shezen E, Hagin D, Ito Y, Takai T, Reich-Zeliger S, et al: Deletion of cognate CD8 T cells by immature dendritic cells: A novel role for perforin, granzyme A, TREM-1, and TLR7. Blood 120: 1647-1657, 2012.

8. Schenk M, Bouchon A, Seibold F and Mueller C: TREM-1-expressing intestinal macrophages crucially amplify chronic inflammation in experimental colitis and inflammatory bowel diseases. J Clin Invest 117: 3097-3106, 2007.

9. Kamei K, Yasuda T, Ueda T, Qiang F, Takeyama Y and Shiozaki H: Role of triggering receptor expressed on myeloid cells-1 in experimental severe acute pancreatitis. J Hepatobiliary Pancreat Sci 17: 305-312, 2010.

10. Liu CL, Hsieh WY, Wu CL, Kuo HT and Lu YT: Triggering receptor expressed on myeloid cells-1 in pleural effusions: A marker of inflammatory disease. Respir Med 101: 903-909, 2007.

11. Kuai J, Gregory B, Hill A, Pittman DD, Feldman JL, Brown T, Carito B, O'Toole M, Ramsey R, Adolfsson O, et al: TREM-1 expression is increased in the synovium of rheumatoid arthritis patients and induces the expression of pro-inflammatory cytokines. Rheumatol 48: 1352-1358, 2009.

12. Schiechl G, Brunner SM, Kesselring R, Martin M, Ruemmele P, Mack M, Hirt SW, Schlitt HJ, Geissler EK and Fichtner-Feigl S: Inhibition of innate co-receptor TREM-1 signaling reduces CD4(+) T cell activation and prolongs cardiac allograft survival. Am J Transplant 13: 1168-1180, 2013.

13. Liao R, Sun TW, Yi Y, Wu H, Li YW, Wang JX, Zhou J, Shi YH, Cheng YF, Qiu SJ and Fan J: Expression of TREM-1 in hepatic stellate cells and prognostic value in hepatitis B-related hepatocellular carcinoma. Cancer Sci 103: 984-992, 2012.

14. Gibot S, Alauzet C, Massin F, Sennoune N, Faure GC, Béné MC, Lozniewski A, Bollaert PE and Lévy B: Modulation of the triggering receptor expressed on myeloid cells-1 pathway during pneumonia in rats. J Infect Dis 194: 975-983, 2006.

15. Guiding principles for the care and use of vertebrate animals in research and training. Am Physiol Soc Counc, 2014.

16. Ramkalawan H, Wang YZ, Hurbungs A, Yang YF, Tian FF, Zhou WB, Li J, Yang H, Xiao B and Zhang W: Pioglitazone, PPAR $\gamma$ agonist, attenuates experimental autoimmune neuritis. Inflammation 35: 1338-1347, 2012.

17. Zhou J, Chai F, Lu G, Hang G, Chen C, Chen X and Shi J: TREM-1 inhibition attenuates inflammation and tumor within the colon. Int Immunopharmacol 17: 155-161, 2013.

18. Sun M, Zhu M, Chen K, Nie X, Deng Q, Hazlett LD, Wu Y, Li M, Wu M and Huang X: TREM-2 promotes host resistance against Pseudomonas aeruginosa infection by suppressing corneal inflammation via a PI3K/Akt signaling pathway. Invest Ophthalmol Vis Sci 54: 3451-3462, 2013.

19. Livak KJ and Schmittgen TD: Analysis of relative gene expression data using real-time quantitative PCR and the 2(-Delta Delta C(T)) Method. Methods 25: 402-408, 2001.

20. Aronovich R, Katzav A and Chapman J: The strategies used for treatment of experimental autoimmune neuritis (EAN): A beneficial effect of glatiramer acetate administered intraperitoneally. Clin Rev Allergy Immunol 42: 181-188, 2012. 
21. Horst SA, Linnér A, Beineke A, Lehne S, Höltje C, Hecht A, Norrby-Teglund A, Medina E and Goldmann O: Prognostic value and therapeutic potential of TREM-1 in Streptococcus pyogenes- induced sepsis. J Innate Immun 5: 581-590, 2013.

22. Wang F, Liu S, Wu S, Zhu Q, Ou G, Liu C, Wang Y, Liao Y and Sun Z: Blocking TREM-1 signaling prolongs survival of mice with Pseudomonas aeruginosa induced sepsis. Cell Immunol 272: 251-258, 2012.

23. Abbott NJ, Rönnbäck L and Hansson E: Astrocyte-endothelia interactions at the blood-brain barrier. Nat Rev Neurosci 7: 41-53, 2006.
24. Zhang HL, Hassan MY, Zheng XY, Azimullah S, Quezada HC, Amir N, Elwasila M, Mix E, Adem A and Zhu J: Attenuated EAN in TNF-alpha deficient mice is associated with an altered balance of M1/M2 macrophages. PLoS One 7: e38157, 2012.

25. Langert KA, Von Zee CL and Stubbs EB Jr: Cdc42 GTPases facilitate TNF-alpha-mediated secretion of CCL2 from peripheral nerve microvascular endoneurial endothelial cells. J Peripher Nerv Syst 18: 199-208, 2013

26. Malemud CJ and Miller AH: Pro-inflammatory cytokine-induced SAPK/MAPK and JAK/STAT in rheumatoid arthritis and the new anti-depression drugs. Expert Opin Ther Targets 12: 171-183, 2008. 